IoAnnis K. Argyros (Lawton, OK)

Santhosh George (Karnataka)

Shobha Monnanda ERAppa (Karnataka)

\title{
LOCAL CONVERGENCE FOR A FAMILY OF ITERATIVE METHODS BASED ON DECOMPOSITION TECHNIQUES
}

Abstract. We present a local convergence analysis for a family of iterative methods obtained by using decomposition techniques. The convergence of these methods was shown before using hypotheses on up to the seventh derivative although only the first derivative appears in these methods. In the present study we expand the applicability of these methods by showing convergence using only the first derivative. Moreover we present a radius of convergence and computable error bounds based only on Lipschitz constants. Numerical examples are also provided.

1. Introduction. In this paper the problem of approximating a locally unique solution $x^{*}$ of the equation

$$
F(x)=0
$$

is analysed. Here $F: D \subseteq X \rightarrow Y$ is a Fréchet-differentiable operator, $X, Y$ are Banach spaces and $D$ is a convex subset of $X$. Newton-like methods are widely used for finding solutions of (1.1), and their convergence is usually studied using semi-local and local convergence analysis. The semilocal convergence analysis is, based on the information around an initial point, to give conditions ensuring the convergence of the iterative procedure; while the local analysis is, based on the information around a solution, to find estimates of the radii of convergence balls [3, 4, 20, 22, 24, 25.

2010 Mathematics Subject Classification: 65D10, 65D99.

Key words and phrases: decomposition techniques, local convergence, order of convergence, radius of convergence.

Received 9 February 2015; revised 30 May 2015.

Published online 2 December 2015. 
Third order methods such as Euler's, Halley's, super Halley's and Chebyshev's methods [1]-33] require the evaluation of the second derivative $F^{\prime \prime}$ at each step, which in general is very expensive. That is why many authors have used higher order multi-point methods [1]-33. In this paper, we introduce the three-step iterative method defined for each $n=0,1,2, \ldots$ by

$$
\begin{aligned}
y_{n} & =x_{n}-\alpha F^{\prime}\left(x_{n}\right)^{-1} F\left(x_{n}\right), \\
z_{n} & =y_{n}-\beta A_{n}^{-1} F\left(y_{n}\right), \\
x_{n+1} & =z_{n}-\gamma B_{n}^{-1} F\left(z_{n}\right),
\end{aligned}
$$

where $x_{0}$ is an initial point, $\alpha, \beta, \gamma \in \mathbb{R}, A_{n}=\sum_{i=1}^{p} w_{i} F^{\prime}\left(x_{n}+\theta_{i}\left(y_{n}-x_{n}\right)\right)$, $B_{n}=\sum_{i=1}^{p} w_{i} F^{\prime}\left(x_{n}+\theta_{i}\left(z_{n}-x_{n}\right)\right), \theta_{i} \in[0,1]$ for $i=1, \ldots, p, p$ is a positive integer and the weights $w_{i} \in S$ satisfy $\sum_{i=1}^{p}\left\|w_{i}\right\|=1$. Method (1.2) reduces to earlier methods resulting from the Adomian decomposition [2], other decompositions [1-3, 23, 27], quadrature formulae and other methods in the special case when $X=Y=\mathbb{R}$.

Let us mention some special cases. Notice that $x_{n}, y_{n}, w_{i}, \theta_{i}, i=1, \ldots, p$, and $F^{\prime}$ define $A_{n}$ and $B_{n}$.

- Noor et al. [25 27] fourth order method: $\alpha=\beta=\gamma=1$, and

$$
\begin{aligned}
y_{n} & =x_{n}-F^{\prime}\left(x_{n}\right)^{-1} F\left(x_{n}\right), \\
z_{n} & =y_{n}-A_{n}^{-1} F\left(y_{n}\right), \\
x_{n+1} & =z_{n}-B_{n}^{-1} F\left(z_{n}\right) .
\end{aligned}
$$

- Noor et al. 25 27] third order method: $\alpha=\beta=1$ and $\gamma=0$, and

$$
\begin{aligned}
y_{n} & =x_{n}-F^{\prime}\left(x_{n}\right)^{-1} F\left(x_{n}\right), \\
x_{n+1} & =y_{n}-A_{n}^{-1} F\left(y_{n}\right) .
\end{aligned}
$$

- Newton's second order method: $\alpha=1$ and $\beta=\gamma=0$, and

$$
x_{n+1}=x_{n}-F^{\prime}\left(x_{n}\right)^{-1} F\left(x_{n}\right)
$$

with efficiency index 1.4142 .

- Two step Newton's third order method considered by Traub [5, 6]: $\alpha=\gamma=p=w_{1}=\theta_{1}=1$ and $\beta=0$, and

$$
\begin{aligned}
y_{n} & =x_{n}-F^{\prime}\left(x_{n}\right)^{-1} F\left(x_{n}\right), \\
x_{n+1} & =y_{n}-F^{\prime}\left(x_{n}\right)^{-1} F\left(y_{n}\right),
\end{aligned}
$$

with efficiency index 1.4422 .

- Midpoint two step Newton's third order method [5, 6, 30, 32]: $\alpha=\beta=$ $p=w_{1}=1, \theta=1 / 2$ and $\gamma=0$, and 


$$
\begin{aligned}
y_{n} & =x_{n}-F^{\prime}\left(x_{n}\right)^{-1} F\left(x_{n}\right), \\
x_{n+1} & =y_{n}-F^{\prime}\left(\frac{x_{n}+y_{n}}{2}\right)^{-1} F\left(y_{n}\right),
\end{aligned}
$$

with efficiency index 1.3161.

- Third order method: $\alpha=\beta=1, \gamma=0, p=2, w_{1}=1 / 4, w_{2}=3 / 4$, $\theta_{1}=0$ and $\theta_{2}=2 / 3$, and

$$
\begin{aligned}
y_{n} & =x_{n}-F^{\prime}\left(x_{n}\right)^{-1} F\left(x_{n}\right), \\
x_{n+1} & =y_{n}-\frac{1}{4}\left(F^{\prime}\left(x_{n}\right)+3 F^{\prime}\left(\frac{x_{n}+2 y_{n}}{3}\right)\right)^{-1} F\left(y_{n}\right),
\end{aligned}
$$

with efficiency index 1.3161.

- Fourth order method: $\alpha=\beta=\gamma=p=1$ and $\theta_{1}=0$, and

$$
\begin{aligned}
y_{n} & =x_{n}-F^{\prime}\left(x_{n}\right)^{-1} F\left(x_{n}\right), \\
z_{n} & =y_{n}-F^{\prime}\left(x_{n}\right)^{-1} F\left(y_{n}\right), \\
x_{n+1} & =z_{n}-F^{\prime}\left(x_{n}\right)^{-1} F\left(z_{n}\right),
\end{aligned}
$$

with efficiency index 1.4142 .

Notice that methods (1.4)- 1.9$)$ are special cases of method (1.3). Many other choices are also possible [5, 6, 29]. Therefore it is important to study these methods in a unified way. A problem with these methods is that they require the existence of the fourth derivative of $F$. This limits the applicability of these methods. As a motivational example, let us define a function $f$ on $D=[-1 / 2,5 / 2]$ by

$$
f(x)= \begin{cases}x^{3} \ln x^{2}+x^{5}-x^{4}, & x \neq 0 \\ 0, & x=0\end{cases}
$$

Choose $x^{*}=1$. We have

$$
\begin{aligned}
f^{\prime}(x) & =3 x^{2} \ln x^{2}+5 x^{4}-4 x^{3}+2 x^{2}, \quad f^{\prime}(1)=3, \\
f^{\prime \prime}(x) & =6 x \ln x^{2}+20 x^{3}-12 x^{2}+10 x, \\
f^{\prime \prime \prime}(x) & =6 \ln x^{2}+60 x^{2}-24 x+22 .
\end{aligned}
$$

Then, obviously, $f^{\prime \prime \prime}$ is unbounded on $D$. In the present paper we only use hypotheses on the first Fréchet derivative. This way we expand the applicability of method 1.2 .

The rest of the paper is organized as follows. The local convergence of method (1.2) is analysed in Section 2, whereas the numerical examples are given in the concluding Section 3.

2. Local convergence analysis. In this section we present the local convergence analysis of method 1.2 . Let $L_{0}, L>0, M \geq 1$ and $\alpha, \beta, \gamma \in \mathbb{R}$ be given parameters. It is convenient for the local convergence analysis of 
method (1.2) to define some scalar functions and parameters. Define functions $g_{1}, f_{2}, h_{f_{2}}$ on the interval $\left[0,1 / L_{0}\right)$ by

$$
\begin{aligned}
g_{1}(t) & =\frac{1}{2\left(1-L_{0} t\right)}(L t+2 M|1-\alpha|), \\
f_{2}(t) & =L_{0} \sum_{i=1}^{p}\left\|w_{i}\right\|\left(\left(1-\theta_{i}\right)+\theta_{i} g_{1}(t)\right) t, \\
h_{f_{2}}(t) & =f_{2}(t)-1,
\end{aligned}
$$

and parameters

$$
r_{1}=\frac{2(1-M|1-\alpha|)}{2 L_{0}+L}, \quad r_{A}=\frac{2}{2 L_{0}+L} .
$$

Suppose that $M|1-\alpha|<1$. Then $g_{1}\left(r_{1}\right)=1$ and $0 \leq g_{1}(t)<1$ for each $t \in\left[0, r_{1}\right)$ and $r_{1}<r_{A}<1 / L_{0}$. We have $h_{f_{2}}(0)=-1<0$ and $h_{f_{2}}(t) \rightarrow \infty$ as $t \rightarrow 1 / L_{0}{ }^{-}$. It follows from the Intermediate Value Theorem that $h_{f_{2}}$ has zeros in $\left(0,1 / L_{0}\right)$. Denote by $r_{f_{2}}$ the smallest such zero. Define functions $g_{2}$ and $h_{2}$ on $\left[0, r_{f_{2}}\right)$ by

$$
g_{2}(t)=\left(1+\frac{|\beta| M}{1-f_{2}(t)}\right) g_{1}(t), \quad h_{2}(t)=g_{2}(t)-1 .
$$

Suppose that $(1+M|\beta|) M|1-\alpha|<1$. Then $h_{2}(0)=(1+M|\beta|) M|1-\alpha|-1$ $<0$ and $h_{2}(t) \rightarrow \infty$ as $t \rightarrow r_{f_{2}}^{-}$. Denote by $r_{2}$ the smallest zero of $h_{2}$ in $\left(0, r_{f_{2}}\right)$. Moreover, define functions $f_{3}, h_{3}$ on $\left[0, r_{2}\right)$ by

$$
f_{3}(t)=L_{0} \sum_{i=1}^{p}\left\|w_{i}\right\|\left(\left(1-\theta_{i}\right)+\theta_{i} g_{2}(t)\right) t, \quad g_{3}(t)=\left(1+\frac{M|\beta|}{1-f_{3}(t)}\right) g_{2}(t)
$$

and $h_{3}(t)=g_{3}(t)-1$. Then again as above we have $f_{3}(0)-1=-1<0$ and $f_{3}(t) \rightarrow \infty$ as $t \rightarrow 1 / L_{0}{ }^{-}$. Hence $f_{3}(t)-1$ has a smallest zero denoted by $r_{3}$ in $\left(0,1 / L_{0}\right)$.

Suppose that

$$
(1+|\beta| M)(1+|\gamma| M \mid) M|1-\alpha|<1 .
$$

Then

$$
h_{3}(0)=(1+M|\beta|)(1+M|\gamma|) M|1-\alpha|-1<0
$$

and

$$
h_{3}\left(r_{2}\right)=\frac{M|\gamma|}{1-f_{3}\left(r_{2}\right)}>0,
$$

since $f_{3}\left(r_{2}\right)<1$. Denote by $\bar{r}_{3}$ the smallest zero of $h_{3}$ in $\left(0, r_{2}\right)$. Define 
$r=\min \left\{r_{1}, r_{2}, \bar{r}_{3}\right\}$. Then for each $t \in[0, r)$,

$$
\begin{array}{ll}
0 \leq g_{1}(t)<1, & \\
0 \leq g_{2}(t)<1, & 0 \leq f_{2}(t)<1, \\
0 \leq g_{3}(t)<1, & 0 \leq f_{3}(t)<1 .
\end{array}
$$

Denote by $U(v, \rho), \bar{U}(v, \rho)$ the open and closed balls in $S$ with center $v \in S$ and of radius $\rho>0$. Next, we present the local convergence analysis of method (1.2) using the preceding notation.

Theorem 2.1. Let $F: D \subset X \rightarrow Y$ be a Fréchet differentiable operator. Suppose that there exist $x^{*} \in D, L_{0}, L>0, M \geq 1$ and $\alpha, \beta, \gamma \in S$ such that for each $x, y \in D$, (2.1) holds and

$$
\begin{aligned}
& F\left(x^{*}\right)=0, \quad F^{\prime}\left(x^{*}\right)^{-1} \in L(Y, X), \\
& \left\|F^{\prime}\left(x^{*}\right)^{-1}\left(F^{\prime}(x)-F^{\prime}\left(x^{*}\right)\right)\right\| \leq L_{0}\left\|x-x^{*}\right\|, \\
& \left\|F^{\prime}\left(x^{*}\right)^{-1}\left(F^{\prime}(x)-F^{\prime}(y)\right)\right\| \leq L\|x-y\|, \\
& \left\|F^{\prime}\left(x^{*}\right)^{-1} F^{\prime}(x)\right\| \leq M,
\end{aligned}
$$

and

$$
\bar{U}\left(x^{*}, r\right) \subseteq D,
$$

where $r$ is defined before Theorem 2.1. Then the sequence $\left\{x_{n}\right\}$ generated for $x_{0} \in U\left(x^{*}, r\right)-\left\{x^{*}\right\}$ by method $(1.2)$ is well defined, remains in $U\left(x^{*}, r\right)$ for each $n=0,1,2, \ldots$ and converges to $x^{*}$. Moreover,

$$
\begin{aligned}
\left\|y_{n}-x^{*}\right\| & \leq g_{1}\left(\left\|x_{n}-x^{*}\right\|\right)\left\|x_{n}-x^{*}\right\|<\left\|x_{n}-x^{*}\right\|<r, \\
\left\|z_{n}-x^{*}\right\| & \leq g_{2}\left(\left\|x_{n}-x^{*}\right\|\right)\left\|x_{n}-x^{*}\right\|<\left\|x_{n}-x^{*}\right\|, \\
\left\|x_{n+1}-x^{*}\right\| & \leq g_{3}\left(\left\|x_{n}-x^{*}\right\|\right)\left\|x_{n}-x^{*}\right\|<\left\|x_{n}-x^{*}\right\|,
\end{aligned}
$$

where the " $g$ " functions are defined before Theorem 2.1. Furthermore, for $T \in\left[r, 2 / L_{0}\right), x^{*}$ is the only solution of the equation $F(x)=0$ in $\bar{U}\left(x^{*}, T\right)$ $\cap D$.

Proof. We shall show 2.10 and (2.12) by induction. Using (2.6), the definition of $r$ and the hypothesis $x_{0} \in U\left(x^{*}, r\right)-\left\{x^{*}\right\}$, we obtain

$$
\left\|F^{\prime}\left(x^{*}\right)^{-1}\left(F^{\prime}\left(x_{0}\right)-F^{\prime}\left(x^{*}\right)\right)\right\| \leq L_{0}\left\|x_{0}-x^{*}\right\|<L_{0} r<1 .
$$

It follows from (2.13) and the Banach Lemma on invertible operators [3, 4, 26, 28, that $F^{\prime}\left(x_{0}\right)^{-1} \in L(Y, X)$ and

$$
\left\|F^{\prime}\left(x_{0}\right)^{-1} F^{\prime}\left(x^{*}\right)\right\| \leq \frac{1}{1-L_{0}\left\|x_{0}-x^{*}\right\|} .
$$

Hence $y_{0}$ is well defined by the first substep of method 1.2 for $n=0$. By 
2.5) we can write

$$
F\left(x_{0}\right)=F\left(x_{0}\right)-F\left(x^{*}\right)=\int_{0}^{1} F^{\prime}\left(x^{*}+\theta\left(x_{0}-x^{*}\right)\right)\left(x_{0}-x^{*}\right) d \theta .
$$

Notice that $\left\|x^{*}+\theta\left(x_{0}-x^{*}\right)-x^{*}\right\|=\theta\left\|x_{0}-x^{*}\right\| \leq\left\|x_{0}-x^{*}\right\|<r$. Hence, $x^{*}+\theta\left(x_{0}-x^{*}\right) \in U\left(x^{*}, r\right)$. Then, by 2.8 and 2.15), we get

$$
\begin{aligned}
\left\|F^{\prime}\left(x^{*}\right)^{-1} F\left(x_{0}\right)\right\| & =\left\|\int_{0}^{1} F^{\prime}\left(x^{*}+\theta\left(x_{0}-x^{*}\right)\right)\left(x_{0}-x^{*}\right) d \theta\right\| \\
& \leq M\left\|x_{0}-x^{*}\right\| .
\end{aligned}
$$

Then it follows from method $(1.2)$ for $n=0,(2.2),(2.5),(2.7),(2.15), 2.16$ and the definition of $r$ that

$$
\begin{aligned}
\leq & \left\|F^{\prime}\left(x_{0}\right)^{-1} F^{\prime}\left(x^{*}\right)\right\|\left\|\int_{0}^{1} F^{\prime}\left(x^{*}\right)^{-1}\left(F^{\prime}\left(x_{0}+\theta\left(x_{0}-x^{*}\right)\right)-F^{\prime}\left(x_{0}\right)\right)\left(x_{0}-x^{*}\right) d \theta\right\| \\
& +|1-\alpha|\left\|F^{\prime}\left(x_{0}\right)^{-1} F^{\prime}\left(x^{*}\right)\right\|\left\|F^{\prime}\left(x^{*}\right)^{-1} F\left(x_{0}\right)\right\| \\
\leq & \frac{L\left\|x_{0}-x^{*}\right\|^{2}}{2\left(1-L_{0}\left\|x_{0}-x^{*}\right\|\right)}+\frac{M\left\|x_{0}-x^{*}\right\|}{1-L_{0}\left\|x_{0}-x^{*}\right\|} \\
= & g_{1}\left(\left\|x_{0}-x^{*}\right\|\right)\left\|x_{0}-x^{*}\right\|<\left\|x_{0}-x^{*}\right\|<r,
\end{aligned}
$$

which shows 2.10 for $n=0$ and $y_{0} \in U\left(x^{*}, r\right)$. We have

$$
\begin{aligned}
\left\|x_{0}+\theta_{i}\left(y_{0}-x_{0}\right)-x^{*}\right\| & \leq\left(1-\theta_{i}\right)\left\|x_{0}-x^{*}\right\|+\theta_{i}\left\|y_{0}-x^{*}\right\| \\
& <\left(1-\theta_{i}\right) r+\theta_{i} r=r .
\end{aligned}
$$

Hence, $x_{0}+\theta_{i}\left(y_{0}-x^{*}\right) \in U\left(x^{*}, r\right)$. We shall show that $A_{0}^{-1} \in L(Y, X)$. Using 2.2, 2.16, 2.17) and the definition of $r$, we obtain

$$
\begin{aligned}
\| F^{\prime}\left(x^{*}\right)^{-1} & \left(A_{0}-F^{\prime}\left(x^{*}\right)\right) \| \\
& \leq \sum_{i=1}^{p}\left\|w_{i}\right\|\left\|F^{\prime}\left(x^{*}\right)^{-1}\left(F^{\prime}\left(x_{0}+\theta_{i}\left(y_{0}-x_{0}\right)\right)-F^{\prime}\left(x^{*}\right)\right)\right\| \\
& \leq L_{0} \sum_{i=1}^{p}\left\|w_{i}\right\|\left(\left(1-\theta_{i}\right)\left\|x_{0}-x^{*}\right\|+\theta_{i}\left\|y_{0}-x^{*}\right\|\right) \\
& \leq L_{0} \sum_{i=1}^{p}\left\|w_{i}\right\|\left(1-\theta_{i}+\theta_{i} g_{1}\left(\left\|x_{0}-x^{*}\right\|\right)\right)\left\|x_{0}-x^{*}\right\| \\
& =f_{2}\left(\left\|x_{0}-x^{*}\right\|\right)<f_{2}(r)<1 .
\end{aligned}
$$


It follows from 2.18 that $A_{0}^{-1} \in L(Y, X)$ and

$$
\left\|A_{0}^{-1} F^{\prime}\left(x^{*}\right)\right\| \leq \frac{1}{1-f_{2}\left(\left\|x_{0}-x^{*}\right\|\right)} .
$$

Hence, $z_{0}$ is well defined by method (1.2) for $n=0$. Then, using (2.2), (2.16) (for $y_{0}=x_{0}$ ), 2.17) and (2.19) we get

$$
\begin{aligned}
\left\|z_{0}-x^{*}\right\| & \leq\left\|y_{0}-x^{*}\right\|+\frac{|\beta| M\left\|y_{0}-x^{*}\right\|}{1-f_{2}\left(\left\|x_{0}-x^{*}\right\|\right)} \\
& =\left(1+\frac{|\beta| M}{1-f_{2}\left(\left\|x_{0}-x^{*}\right\|\right)}\right)\left(\left\|y_{0}-x^{*}\right\|\right) \\
& \leq\left(1+\frac{|\beta| M}{1-f_{2}\left(\left\|x_{0}-x^{*}\right\|\right)}\right) g_{1}\left(\left\|x_{0}-x^{*}\right\|\right)\left\|x_{0}-x^{*}\right\| \\
& =g_{2}\left(\left\|x_{0}-x^{*}\right\|\right)\left\|x_{0}-x^{*}\right\|<\left\|x_{0}-x^{*}\right\|<r,
\end{aligned}
$$

which shows (2.11) for $n=0$ and $z_{0} \in U\left(x^{*}, r\right)$.

Next we show that $B_{0}^{-1} \in L(Y, X)$. Let $z_{0}=y_{0}, g_{1}=g_{2}$, and $f_{2}=f_{3}$, in (2.18). Then by (2.4) we obtain

$$
\left\|F^{\prime}\left(x^{*}\right)^{-1}\left(B_{0}-F^{\prime}\left(x^{*}\right)\right)\right\| \leq f_{3}\left(\left\|x_{0}-x^{*}\right\|\right)<f_{3}(r)<1 .
$$

It follows that $B_{0}^{-1} \in L(Y, X)$ and

$$
\left\|B_{0}^{-1} F^{\prime}\left(x^{*}\right)\right\| \leq \frac{1}{1-f_{3}\left(\left\|x_{0}-x^{*}\right\|\right)} .
$$

Hence, $x_{1}$ is well defined by the third substep of method $(1.2)$ for $n=0$. Then, using (2.4), 2.16) (for $z_{0}=x_{0}$ ), 2.20) and (2.22), we get

$$
\begin{aligned}
\left\|x_{1}-x^{*}\right\| & \leq\left\|z_{0}-x^{*}\right\|+\frac{|\gamma| M\left\|z_{0}-x^{*}\right\|}{1-f_{3}\left(\left\|x_{0}-x^{*}\right\|\right)} \\
& =\left(1+\frac{|\gamma| M}{1-f_{3}\left(\left\|x_{0}-x^{*}\right\|\right)}\right)\left(\left\|z_{0}-x^{*}\right\|\right) \\
& \leq\left(1+\frac{|\gamma| M}{1-f_{3}\left(\left\|x_{0}-x^{*}\right\|\right)}\right) g_{2}\left(\left\|x_{0}-x^{*}\right\|\right)\left\|x_{0}-x^{*}\right\| \\
& =g_{3}\left(\left\|x_{0}-x^{*}\right\|\right)\left\|x_{0}-x^{*}\right\|<\left\|x_{0}-x^{*}\right\|<r,
\end{aligned}
$$

which shows 2.12 for $n=0$ and $x_{1} \in U\left(x^{*}, r\right)$. Hence by simply replacing $x_{0}, y_{0}, z_{0}, x_{1}$ by $x_{k}, y_{k}, z_{k}, x_{k+1}$ in the preceding estimates we arrive at estimates (2.10)-(2.12). Using the estimate $\left|x_{k+1}-x^{*}\right|<\left|x_{k}-x^{*}\right|<r$, we deduce that $x_{k+1} \in U\left(x^{*}, r\right)$ and $\lim _{k \rightarrow \infty} x_{k}=x^{*}$.

To prove the uniqueness part, let $Q=\int_{0}^{1} F^{\prime}\left(y^{*}+\theta\left(x^{*}-y^{*}\right)\right) d \theta$ for some $y^{*} \in \bar{U}\left(x^{*}, T\right)$ with $F\left(y^{*}\right)=0$. Using (2.6) we get 


$$
\begin{aligned}
\left|F^{\prime}\left(x^{*}\right)^{-1}\left(Q-F^{\prime}\left(x^{*}\right)\right)\right| & \leq \int_{0}^{1} L_{0}\left|y^{*}+\theta\left(x^{*}-y^{*}\right)-x^{*}\right| d \theta \\
& \leq L_{0} \int_{0}^{1}(1-\theta)\left|x^{*}-y^{*}\right| d \theta \leq \frac{L_{0}}{2} T<1 .
\end{aligned}
$$

It follows from (2.24) and the Banach Lemma on invertible functions that $Q$ is invertible. Finally, from the identity $0=F\left(x^{*}\right)-F\left(y^{*}\right)=Q\left(x^{*}-y^{*}\right)$, we deduce that $x^{*}=y^{*}$.

REMARK 2.2. 1. In view of (2.6) and the estimate

$$
\begin{aligned}
\left\|F^{\prime}\left(x^{*}\right)^{-1} F^{\prime}(x)\right\| & =\left\|F^{\prime}\left(x^{*}\right)^{-1}\left(F^{\prime}(x)-F^{\prime}\left(x^{*}\right)\right)+I\right\| \\
& \leq 1+\left\|F^{\prime}\left(x^{*}\right)^{-1}\left(F^{\prime}(x)-F^{\prime}\left(x^{*}\right)\right)\right\| \leq 1+L_{0}\left\|x-x^{*}\right\|
\end{aligned}
$$

condition (2.8) can be dropped and $M$ can be replaced by

$$
M(t)=1+L_{0} t
$$

or $M(t)=M=2$, since $t \in\left[0,1 / L_{0}\right)$.

2. The results obtained here can be used for operators $F$ satisfying autonomous differential equations [3] of the form

$$
F^{\prime}(x)=P(F(x))
$$

where $P$ is a continuous operator. Then, since $F^{\prime}\left(x^{*}\right)=P\left(F\left(x^{*}\right)\right)=P(0)$, we can apply the results without actually knowing $x^{*}$. For example, let $F(x)=e^{x}-1$. Then we can choose $P(x)=x+1$.

3 . The radius $r_{A}$ was shown to be the convergence radius of Newton's method [5, 6]

$$
x_{n+1}=x_{n}-F^{\prime}\left(x_{n}\right)^{-1} F\left(x_{n}\right) \quad \text { for } n=0,1,2, \ldots,
$$

under the conditions (2.6) and 2.7). It follows from the definition of $r$ that the convergence radius $r$ of method $(1.2)$ cannot be larger than the convergence radius $r_{A}$ of the second order Newton's method (2.25). As already noted in [5, 6], $r_{A}$ is at least as large as the convergence ball given by Rheinboldt 31

$$
r_{R}=\frac{2}{3 L} .
$$

In particular, for $L_{0}<L$ we have

$$
r_{R}<r
$$

and

$$
\frac{r_{R}}{r_{A}} \rightarrow \frac{1}{3} \quad \text { as } \frac{L_{0}}{L} \rightarrow 0 .
$$

That is, our convergence ball $r_{A}$ is at most three times larger than Rheinboldt's. The same value for $r_{R}$ was given by Traub [32]. 
4. It is worth noticing that method (1.2) does not change when we use the conditions of Theorem 2.1 instead of the stronger conditions used in [1, 2, 3, 25, 26, 27. Moreover, we can compute the computational order of convergence (COC) [15] defined by

$$
\xi=\ln \left(\frac{\left\|x_{n+1}-x^{*}\right\|}{\left\|x_{n}-x^{*}\right\|}\right) / \ln \left(\frac{\left\|x_{n}-x^{*}\right\|}{\left\|x_{n-1}-x^{*}\right\|}\right)
$$

or the approximate computational order of convergence [15]:

$$
\xi_{1}=\ln \left(\frac{\left\|x_{n+1}-x_{n}\right\|}{\left\|x_{n}-x_{n-1}\right\|}\right) / \ln \left(\frac{\left\|x_{n}-x_{n-1}\right\|}{\left\|x_{n-1}-x_{n-2}\right\|}\right) .
$$

This way we obtain in practice the order of convergence in a way that avoids the bounds involving estimates of higher Fréchet derivatives of $F$.

3. Numerical examples. In this section we present two numerical examples. In both, we have taken the parameters $\alpha, \beta, \gamma, w_{i}, \theta_{i}, p, i=1, \ldots, p$, as given in the introduction for method (1.6)-(1.8). We have taken $w_{1}=\theta_{1}=$ $p=1$ for methods 1.3 -1.5 and $p=w_{1}=1$ and $\theta_{1}=0$ for method (1.9).

Example 3.1. Let $X=Y=\mathbb{R}^{3}, D=\bar{U}(0,1), x^{*}=(0,0,0)^{T}$. Define a function $F$ on $D$ for $w=(x, y, z)^{T}$ by

$$
F(w)=\left(e^{x}-1, \frac{e-1}{2} y^{2}+y, z\right)^{T} .
$$

Table 1. Parameters of methods (1.3)-(1.9): Example 3.1

\begin{tabular}{|l|l|l|l|l|l|l|}
\hline $\begin{array}{l}\text { parameters/ } \\
\text { methods }\end{array}$ & $r=\bar{r}_{3}$ & $r_{2}$ & $r_{1}$ & $r_{3}$ & $r_{f_{2}}$ & $\xi$ \\
\hline$(1.3)$ & 0.0667 & 0.1650 & 0.3249 & 0.1490 & 0.3828 & 0.993076 \\
$(1.4)$ & 0.0667 & 0.1650 & 0.3249 & 0.1490 & 0.3828 & 3.655630 \\
$(1.5)$ & 0.2962 & 0.3249 & 0.3249 & 0.3142 & 0.4079 & 3.655630 \\
$(1.6)$ & 0.0667 & 0.1650 & 0.3249 & 0.1490 & 0.3828 & 1.753711 \\
$(1.7)$ & 0.0636 & 0.1570 & 0.3249 & 0.3269 & 0.4079 & 1.994778 \\
$(1.8)$ & 0.0291 & 0.3249 & 0.3249 & 0.3254 & 0.4079 & 2.004979 \\
$(1.9)$ & 0.0667 & 0.1650 & 0.3249 & 0.1490 & 0.3828 & 3.639839 \\
\hline
\end{tabular}

Then the Fréchet derivative is given by

$$
F^{\prime}(v)=\left[\begin{array}{ccc}
e^{x} & 0 & 0 \\
0 & (e-1) y+1 & 0 \\
0 & 0 & 1
\end{array}\right] .
$$

We have $L_{0}=e-1, L=e, M=2$. The parameters are given in Table 1 . 
EXAMPLE 3.2. Returning to the motivational example in the introduction, we have $L_{0}=L=146.6629073, M=2$. The parameters are given in Table 2.

Table 2. Parameters of methods (1.3)-(1.9): Example 3.2

\begin{tabular}{|l|l|l|l|l|l|l|}
\hline $\begin{array}{l}\text { parameters } / \\
\text { methods }\end{array}$ & $r=\bar{r}_{3}$ & $r_{2}$ & $r_{1}$ & $r_{3}$ & $r_{f_{2}}$ & $\xi$ \\
\hline$(1.3)$ & 0.0011 & 0.0026 & 0.0045 & 0.0048 & 0.0050 & 1.002163 \\
$(1.4)$ & 0.0011 & 0.0026 & 0.0045 & 0.0048 & 0.0050 & 0.993283 \\
$(1.5)$ & 0.0036 & 0.0045 & 0.0045 & 0.0042 & 0.0052 & 0.991749 \\
$(1.6)$ & 0.0011 & 0.0026 & 0.0045 & 0.0048 & 0.0050 & 0.992117 \\
$(1.7)$ & 0.0011 & 0.0024 & 0.0045 & 0.0048 & 0.0052 & 0.998581 \\
$(1.8)$ & 0.0003 & 0.0024 & 0.0045 & 0.0048 & 0.0052 & 0.992479 \\
$(1.9)$ & 0.0011 & 0.0026 & 0.0045 & 0.0048 & 0.0050 & 0.992479 \\
\hline
\end{tabular}

\section{References}

[1] S. Abbasbandy, Improving Newton-Raphson method for nonlinear equations by modified Adomian decomposition method, Appl. Math. Comput. 145 (2003), 887-893.

[2] G. Adomian, Solving Frontier Problems of Physics: The Decomposition Method, Kluwer, Dordrecht, 1994.

[3] F. Ahmad, S. Hussain, N. A. Mir and A. Rafiq, New sixth order Jarratt method for solving nonlinear equations, Int. J. Appl. Math. Mech. 5 (2009), 27-35.

[4] S. Amat, M. A. Hernández and N. Romero, A modified Chebyshev's iterative method with at least sixth order of convergence, Appl. Math. Comput. 206 (2008), 164-174.

[5] I. K. Argyros, Convergence and Application of Newton-Type Iterations, Springer, 2008.

[6] I. K. Argyros and S. Hilout, A convergence analysis for directional two-step Newton methods, Numer. Algorithms 55 (2010), 503-528.

[7] S. Bhalekar and V. Daftardar-Gejji, Convergence of the new iterative method, Int. J. Differential Equations 2011, art. ID 989065, 10 pp.

[8] D. D. Bruns and J. E. Bailey, Nonlinear feedback control for operating a nonisothermal CSTR near an unstable steady state, Chem. Engrg. Sci. 32 (1977), 257-264.

[9] V. Candela and A. Marquina, Recurrence relations for rational cubic methods I: The Halley method, Computing 44 (1990), 169-184.

[10] V. Candela and A. Marquina, Recurrence relations for rational cubic methods II: The Chebyshev method, Computing 45 (1990), 355-367.

[11] C. Chun, Some improvements of Jarratt's method with sixth-order convergence, Appl. Math. Comput. 190 (1990), 1432-1437.

[12] C. Chun, Iterative methods improving Newton's method by the decomposition method, Comput. Math. Appl. 50 (2005), 1559-1568.

[13] V. Daftardar-Gejji and H. Jafari, An iterative method for solving non-linear functional equations, J. Math. Anal. Appl. 316 (2006), 753-763.

[14] J. A. Ezquerro and M. A. Hernández, Recurrence relations for Chebyshev-type methods, Appl. Math. Optim. 41 (2000), 227-236.

[15] J. A. Ezquerro and M. A. Hernández, New iterations of R-order four with reduced computational cost, BIT Numer. Math. 49 (2009), 325-342. 
[16] J. A. Ezquerro and M. A. Hernández, On the R-order of the Halley method, J. Math. Anal. Appl. 303 (2005), 591-601.

[17] J. M. Gutiérrez and M. A. Hernández, Recurrence relations for the super-Halley method, Comput. Math. Appl. 36 (1998), 1-8.

[18] M. Ganesh and M. C. Joshi, Numerical solvability of Hammerstein integral equations of mixed type, IMA J. Numer. Anal. 11 (1991), 21-31.

[19] J. H. He, A new iteration method for solving algebraic equations, Appl. Math. Comput. 135 (2003), 81-84.

[20] M. A. Hernández, Chebyshev's approximation algorithms and applications, Comput. Math. Appl. 41 (2001), 433-455.

[21] M. A. Hernández and M. A. Salanova, Sufficient conditions for semilocal convergence of a fourth order multipoint iterative method for solving equations in Banach spaces, Southwest J. Pure Appl. Math. 1999, 29-40.

[22] L. V. Kantorovich and G. P. Akilov, Functional Analysis, Pergamon Press, Oxford, 1982.

[23] Á. A. Magreñán, Different anomalies in a Jarratt family of iterative root-finding methods, Appl. Math. Comput. 233 (2014), 29-38.

[24] Á. A. Magreñán, A new tool to study real dynamics: The convergence plane, Appl. Math. Comput. 248 (2014), 215-224.

[25] M. A. Noor, Some iterative methods for solving non-linear equations using homotopy perturbation method, Int. J. Comput. Math. 87 (2010), 141-149.

[26] M. A. Noor and K. I. Noor, Three-step iterative methods for non-linear equations, Appl. Math. Comput. 183 (2006), 322-327.

[27] M. A. Noor, M. Waseen, K. I. Noor and M. A. Al, New iterative techniques for solving non-linear equations, Appl. Math. Comput. 265 (2015), 1115-1125.

[28] S. K. Parhi and D. K. Gupta, Semilocal convergence of a Stirling-like method in Banach spaces, Int. J. Comput. Methods 7 (2010), 215-228.

[29] M. S. Petković, B. Neta, L. Petković and J. Džunić, Multipoint Methods for Solving Nonlinear Equations, Elsevier, 2013.

[30] H. Ren, Q. Wu and W. Bi, New variants of Jarratt method with sixth-order convergence, Numer. Algorithms 52 (2009), 585-603.

[31] W. C. Rheinboldt, An adaptive continuation process for solving systems of nonlinear equations, in: Mathematical Models and Numerical Methods, A. N. Tikhonov et al. (eds.), Banach Center Publ. 3, PWN, Warszawa, 1978, 129-142.

[32] J. F. Traub, Iterative Methods for the Solution of Equations, Prentice-Hall, Englewood Cliffs, NJ, 1964.

[33] X. Wang and J. Kou, Semilocal convergence of a class of modified super-Halley methods in Banach spaces, J. Optim. Theory. Appl. 153 (2012), 779-793.

Ioannis K. Argyros

Department of Mathematical Sciences

Cameron University

Lawton, OK 73505, U.S.A.

E-mail: iargyros@cameron.edu
Santhosh George, Shobha Monnanda Erappa Department of Mathematical and Computational Sciences NIT Karnataka Karnataka, India 575025 E-mail: sgeorge@nitk.ac.in shobha.me@gmail.com 
